\title{
VARIAÇÕES NA QUALIDADE DE TORAS DE Toona ciliata M. Roem. COM DEZOITO ANOS DE IDADE
}

\author{
Otávio Augusto Carvalho Nassur' ${ }^{1}$ Lucas Rodrigues Rosado², \\ Sebastião Carlos da Silva Rosado ${ }^{3}$, Pablo Marcílio de Carvalho ${ }^{1}$
}

(recebido: 9 de dezembro de 2010; aceito: 28 de setembro de 2012)

\begin{abstract}
RESUMO: A família meliácea contém as mais valiosas espécies produtoras de madeira na região tropical, no entanto o cultivo dessas espécies em sua área de ocorrência natural é limitado, em decorrência do ataque de brocas do gênero Hypsipyla. Em contrapartida, plantios exóticos de cedro australiano (T. ciliata) no Brasil tem tido grande sucesso especialmente pela resistência ao ataque da broca dos cedros, e também por apresentar bom crescimento vegetativo e adaptação ao solo e ao clima. No entanto, a maioria dos plantios comerciais existentes de $T$. ciliata é ainda recente, pouco se sabe a respeito da qualidade das toras que serão destinadas às indústrias do setor madeireiro. Nesse sentido, o presente trabalho almeja qualificar toras de árvores crescidas em plantações com 18 anos e com dimensões apropriadas para o desdobro. A partir desses dados, será possível avaliar a variabilidade entre árvores para definir estratégias de manejo florestal e melhoramento genético para a espécie. Para o presente estudo, foram utilizadas árvores provenientes da cidade de Marechal Floriano - ES, com dezoito anos de idade. As toras foram previamente medidas e, posteriormente, foram avaliados os defeitos, a partir dos mesmos procedeu-se a classificação das árvores. A classificação mostrou que o defeito mais expressivo foi a presença de nós e protuberâncias, em contrapartida o defeito menos pronunciado foi a conicidade das toras. As árvores apresentaram expressivas variações quanto aos defeitos, sugerindo a necessidade de condução de programas de melhoramento genético em associação com práticas adequadas de manejo florestal.
\end{abstract}

Palavras-chave: Defeitos em toras, cedro australiano, melhoramento florestal.

\section{VARIATIONS IN LOG QUALITY OF EIGHTEEN YEARS OLD Toona ciliata M. Roem.}

\begin{abstract}
The family Meliaceae contains the most valuable timber species found in tropical regions. However, plantation of such species in their natural range is limited by damages from shoot borers of the genus Hypsipyla. Nevertheless, specifically in Brazil, the commercial cultivation of Australian cedar (Toona ciliata) has been successful for presenting a satisfactory vegetative growth, adapting to the Brazilian soil and climate, but especially for being resistant to the cedar shoot borer attacks. In spite of these favorable conditions, the majority of existing T.ciliata commercial plantations is still recent and little is known about the log quality destined for timber industries. In this sense, the present work aims at qualifying logs of 18 years old trees, with dimensions fit for sawing and grown on plantations for timber industries. From the data gathered it will be possible to assess the variability between trees in order to define forest management strategies and of breed improvements for the species. It was used eighteen years old trees from the city of Marechal Floriano - Espírito Santo state. The logs were previously measured and their defects were evaluated afterwards and from which, the trees were classified. The classification showed that the most significant defects were knots and bumps and the least pronounced defect was the log taper. The trees showed significant variations regarding the defects, suggesting the need for breeding programs in association with improved forest management practices.
\end{abstract}

Key words: Defects in logs, Australian cedar, forest improvement

\section{INTRODUÇÃO}

O mogno e os cedros (Cedrela, spp.) têm ocorrência natural, predominantemente, na América Central e do Sul, apresentam altas taxa de crescimento. Infelizmente, quando em monocultivos nas áreas de ocorrência natural, elas sofrem intensos ataques da lagarta Hypsipyla grandella (Lepidoptera: Pyralideae), reduzindo drasticamente a taxa de crescimento das árvores e prejudicando a forma comercial do fuste, uma vez que o inseto destrói o ápice do tronco e ramos e, consequentemente, provocam a perda da dominância apical. $O$ controle da referida lagarta, seja por meio de seleções de genótipos resistentes, controles químicos ou biológicos, até o presente momento, não foi bem sucedido, gerando inseguranças de ordem técnica e econômica nas atividades de reflorestamento.

${ }^{1}$ Engenheiro Florestal, Mestre em Ciência e Tecnologia da Madeira - Universidade Federal de Lavras/UFLA - Departamento de Ciências Florestais - Cx. P. 3037 - 37200-000 - Lavras, MG, Brasil - otaviofacao@ hotmail.com, pablo.pecanha@hotmail.com

${ }^{2}$ Estudante de Engenharia Florestal - Universidade Federal de Lavras/UFLA - Departamento de Ciências Florestais - Cx. P. 3037 37200-000 - Lavras, MG, Brasil - lucasrosado@yahoo.com.br

${ }^{3}$ Engenheiro Florestal, Professor Doutor em Ciências Florestais - Universidade Federal de Lavras/UFLA - Departamento de Ciências Florestais - Cx. P. 3037 - 37200-000 - Lavras, MG, Brasil - scrosado@dcf.ufla.br 
De forma análoga, a Toona ciliata que tem ocorrência natural, predominante, na Ásia e Austrália apresenta situações semelhantes às do mogno e cedros, quando cultivados em áreas de ocorrência natural. A diferença básica surge do fato de que a praga que promove a perda da dominância apical é a $H$. robusta (GRIFFTHS, 2000).

$\mathrm{Na}$ América do Sul, o cultivo da Toona ciliata vem sendo amplamente difundido, em razão dos excelentes resultados obtidos em termos de crescimento vegetativo e adaptação às condições de solo e clima nas diferentes regiões. As árvores apresentam um tronco que é, predominantemente, monopodial e sem a perda da dominância apical. Até o presente momento, não há referências de ataques da $H$. grandella e da ocorrência da H. robusta nas Américas (BYGRAVE; BYGRAVE, 2005) mesmo em condições de monocultivo. Além da ausência dos ataques, outra característica relevante, diz respeito à capacidade da T. ciliata tolerar à sombra, particularidade esta que implica em diversas vantagens, principalmente para produtores que desejam investir em plantios consorciados. Deve-se ressaltar que plantios mistos são menos suscetíveis a ataques de fungos e insetos do que as monoculturas (BRISTOW et al., 2005).

$\mathrm{Na}$ obtenção de madeira serrada de alta qualidade, várias estratégias devem ser utilizadas em conjunto, envolvendo etapas iniciadas no plantio até o processamento final da madeira. O rendimento de madeira serrada está diretamente relacionado com as características da matériaprima utilizada. Toras mais tortuosas ou de formato irregular são potencialmente geradoras de maior quantidade de resíduos, dentre as principais características responsáveis pela perda de rendimento estão a medula excêntrica, grande quantidade de nós, conicidade e bifurcação (ROCHA, 2001).

A conicidade se caracteriza pela diminuição do diâmetro da base para a copa da árvore, sendo mais pronunciada em toras oriundas da parte basal. É uma característica natural de cada espécie, tende a diminuir com a idade da planta e sofre pouca influência genética. A conicidade apresenta-se mais pronunciada em árvores isoladas ou situadas às margens de povoamentos (PURNELL, 1988 citado por SCANAVACA JÚNIOR; GARCIA, 2003; TIBÚRCIO, 2000). Segundo Grosser (1980), a conicidade influencia o rendimento em madeira serrada somente quando apresentar valores superiores a $1 \mathrm{~cm} / \mathrm{m}$ para o diâmetro ou $3 \mathrm{~cm} / \mathrm{m}$ para a circunferência da tora.

Encurvamentos são defeitos na forma do tronco, caracterizando-se por um desvio permanente do eixo do fuste sendo, na maioria dos casos, sob a forma de curvas.
Os encurvamentos tanto podem surgir de fatores genéticos, características da própria espécie, ou serem ocasionadas por influências externas. Os prejuízos causados dependem da forma e quantidade de curvaturas existentes. Esse tipo de defeito repercute tanto no aproveitamento longitudinal como transversal, conduzindo a obtenção de pequenas peças ou excessivo seccionamento das toras (INSTITUTO BRASILEIRO DE DESENVOLVMENTOFLORESTAL- IBDF, 1984).

Na obtenção de produtos de madeira serrada, o diâmetro dos nós assume uma importância decisiva, é possível manter o núcleo nodoso dentro de um diâmetro reduzido, aumentando-se a proporção de madeira com ausência de nós por meio da prática de poda ou desrama (TIBÚRCIO, 2000). Para Hawley e Smith (1972) a presença de nós e a inclinação da grã são os fatores que mais reduzem o valor e a utilidade da madeira em povoamentos manejados. De acordo com Caixeta et al. (2002), defeitos de formação, como a presença de nós, veios de quino, a conicidade e o achatamento, podem ser evitados com a melhoria dos tratos silviculturais. Outro defeito comum em toras é o aparecimento de rachaduras de extremidade, geralmente relacionadas às tensões de crescimento.

A seleção e classificação de árvores (fenótipos) superiores, que forneçam material com baixa intensidade de defeitos devem ser estimuladas nos programas de melhoramento, uma vez que a maioria dessas características apresenta elevada herdabilidade, o que poderá propiciar ganhos consideráveis (CAIXETA et al., 2002). De maneira semelhante, a identificação dos defeitos mais frequentes em toras de determinada espécie, facilita a adoção de práticas adequadas de manejo florestal visando à obtenção de matéria-prima de qualidade superior.

Por ser uma espécie de introdução recente no Brasil, não são relatados estudos a respeito dos defeitos que ocorrem nas toras das árvores de T. ciliata, sobretudo quando produzidas em plantios comerciais com finalidade industrial. Dessa forma, para o estabelecimento de plantações em condições brasileiras, torna-se necessário qualificar a madeira na idade de seu uso industrial. A partir disso, será possível aprimorar as etapas de processamento e, finalmente, definir estratégias de melhoramento genético que, para as condições brasileiras, ainda não foram devidamente implementadas.

Neste trabalho, objetivou-se avaliar as características de toras de árvores de Toona ciliata com 18 anos de idade em relação aos principais defeitos que afetam o rendimento de madeira serrada. 


\section{MATERIAL E MÉTODOS}

\subsection{Amostragem}

Foram abatidas de forma aleatória 21 árvores (origem desconhecida) em plantio localizado no município de Marechal Floriano - ES, de coordenadas aproximadas

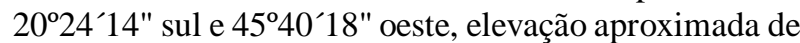
$530 \mathrm{~m}$, e precipitação média anual de $1572 \mathrm{~mm}$ segundo a Agência Nacional de Águas - ANA (2010). O plantio não possuía espaçamento fixo, as árvores não sofreram tratos culturais tais como poda e adubação, durante o seu desenvolvimento.

As árvores foram seccionadas em toras com comprimento entre 2,30 a 3,30 metros e rotuladas. Em seguida, foram transportadas para Unidade Experimental de Desdobro e Secagem da Madeira (DCF-UFLA).

Na sequência é apresentada uma foto (Figura 1) das toras chegando à Unidade Experimental de Desdobro e Secagem da Madeira.

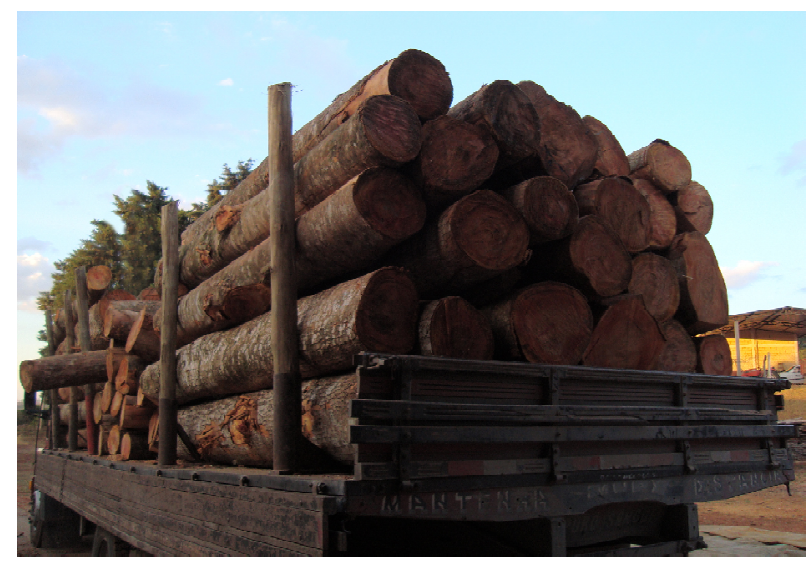

Figura 1 - Toras chegando à unidade experimental de desdobro e secagem da madeira.

Figure 1 - Logs arriving at the wood experimental unity for sawing and drying.

\subsection{Classificação das toras}

Antes de serem desdobradas, as toras ficaram armazenadas no pátio (Figura 2), objetivando a classificação, foram realizadas as seguintes medições:

- Diâmetro, duas medidas no topo e duas na base.

- Comprimento da tora.

- Flecha e o comprimento da parte da tora atingido pelo encurvamento.

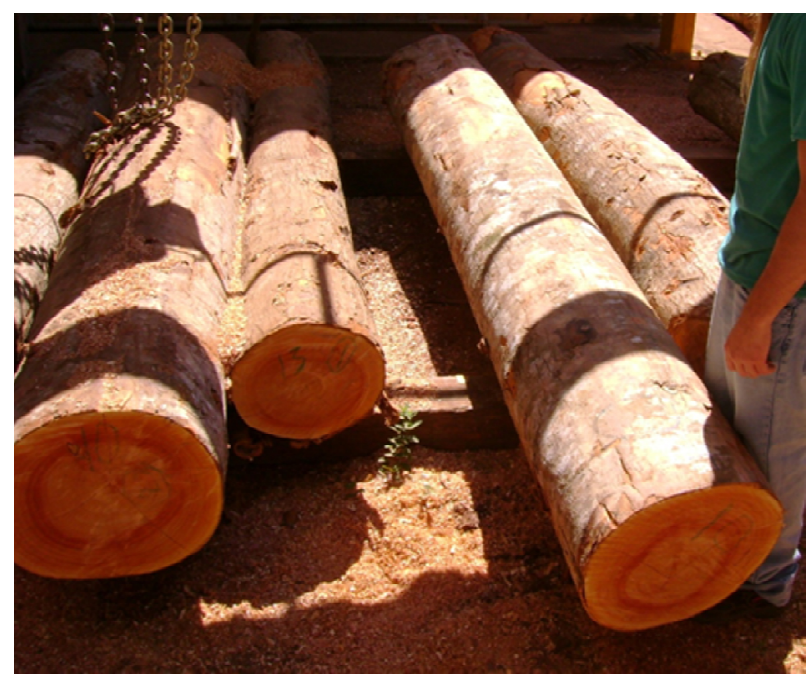

Figura 2 - Toras armazenadas no local de medição.

Figure 2 - Stored logs ready for measurement.

- Desvio de grã.

- Comprimento das rachaduras que atingem a base e o topo.

- Diâmetro de nós e protuberâncias.

- Espessura da maior rachadura.

Com posse dos dados foram obtidos o volume, achatamento, conicidade, encurvamento, inclinação da grã, rachadura de superfície, nós e protuberâncias conforme a norma para medição e classificação de toras de madeiras de folhosas (IBDF, 1984).

Visando a facilitar a comparação e interpretação dos resultados entre as árvores, a classificação foi realizada em função das médias dos defeitos obtida para cada árvore e não pelas toras individuais dessas árvores, estas foram qualificadas em uma das classes, superior (SU), primeira classe (I), segunda classe (II), terceira classe (III) e quarta classe (IV) em relação à forma e à sua superfície rolante, conforme é descrito nos tópicos seguintes.

\subsubsection{Classificação quanto a forma}

Foram considerados, simultaneamente, o achatamento a conicidade e o encurvamento dentro dos critérios mostrados na Tabela 1.

\subsubsection{Classificação quanto a superfície rolante}

Foram consideradas, simultaneamente, as características, inclinação da grã, as rachaduras de superfície e nós e protuberâncias de acordo com os critérios apresentados na Tabela 2.

Cerne, Lavras, v. 19, n. 1, p. 43-49, jan./mar. 2013 
Tabela 1 - Critérios para classificação das árvores quanto a sua forma, adaptado do IBDF (1984).

Table 1 - Criteria for shape classification, adapted from IBDF (1984).

\begin{tabular}{lccccc}
\hline \multirow{2}{*}{ Item } & \multicolumn{5}{c}{ Classes } \\
\cline { 2 - 6 } & $\mathrm{SU}$ & $\mathrm{I}$ & $\mathrm{II}$ & $\mathrm{III}$ & IV \\
\hline Achatamento & $\geq 90 \%$ & $\geq 80 \%$ & $\geq 70 \%$ & $\geq 60 \%$ & n.e \\
Conicidade & $\leq 3 \%$ & $\leq 3 \%$ & $\leq 4 \%$ & n.e & n.e \\
Encurvamento & $\leq 5 \%$ & $\leq 5 \%$ & $\leq 8 \%$ & $\leq 8 \%$ & n.e \\
\hline
\end{tabular}

n.e - não especificado

Tabela 2 - Critérios para classificação das árvores quanto a sua superfície rolante, adaptado do IBDF (1984).

Table 2 - Criteria for the classification of tree surface, adapted from IBDF (1984).

\begin{tabular}{lccccc}
\hline & \multicolumn{5}{c}{ Classes } \\
\cline { 2 - 6 } Item & SU & I & II & III & IV \\
\hline $\begin{array}{l}\text { Inclinação } \\
\text { da grã }\end{array}$ & $\leq 3 \%$ & $\leq 3 \%$ & $\leq 10 \%$ & $\geq 20 \%$ & n.e \\
$\begin{array}{l}\text { Rachaduras } \\
\text { de superfície }\end{array}$ & $\leq 5 \%$ & $\leq 10 \%$ & $\leq 20 \%$ & $\leq 30 \%$ & $\leq 40 \%$ \\
$\begin{array}{l}\text { Nós e } \\
\text { protuberancias }\end{array}$ & $\leq 3 \%$ & $\leq 3 \%$ & $\leq 6 \%$ & $\leq 10 \%$ & $\leq 15 \%$ \\
\hline
\end{tabular}

n.e - não especificado

\section{RESULTADOS E DISCUSSÃO}

As médias e os respectivos coeficientes de variação para achatamento, conicidade e encurvamento estão expressos na Tabela 3.

De acordo com as normas do IBDF (1984) que classifica as toras pelas suas características, são consideradas como de forma superior àquelas que apresentam achatamento igual ou superior a $90 \%$, conicidade igual ou inferior a $3 \%$ e encurvamento igual ou inferior a $5 \%$. Considerando essas três características de qualidade simultaneamente, somente as árvores de número 1, 13 e 14 produzem toras, cujos valores médios as classificam como superiores. Nessa mesma metodologia de classificação, todas as outras árvores são classificadas na classe I.

Segundo Scanavaca Júnior e Garcia (2003), quando a árvore é jovem ela é mais cônica e se torna mais cilíndrica com o aumento da idade. Nesse contexto, e considerando os dados apresentados na Tabela 3 , pode-se supor que árvores de $T$. ciliata na idade analisada de 18 anos já apresentam padrões de forma dentro de limites considerados como sendo superiores ou de primeira classe. Essas considerações têm importância de ordem prática no contexto do manejo florestal, envolvendo práticas de desbaste, ou de corte final.

Do ponto de vista do melhoramento genético, visando à melhoria da forma das árvores e, consequentemente, do rendimento de desdobro da madeira deve se considerar a idade de 18 , ou superior, como ideais para seleção. Outros estudos com idade de avaliações em árvores mais jovens poderiam indicar a realização de seleções genéticas mais precoces.

Comparando os valores de conicidade, apresentados para Eucalyptus, os quais são classificados de forma superior por Evangelista (2007), Ferreira et al. (2004), Scanavaca Júnior e Garcia (2003) e Vale et al. (2002), com os valores de T. ciliata apresentados na Tabela 3, pode-se verificar que várias árvores já se classificam na referida categoria de forma. Considerando que a população de $T$. ciliata estudada não foi manejada e nem geneticamente melhorada, pode-se esperar que práticas adequadas de manejo e de seleção podem reduzir a conicidade das árvores, tornando-as mais apropriadas para a utilização industrial.

Na Tabela 4, são apresentados os valores obtidos para nós e protuberâncias, rachaduras superficiais, e inclinação da grã. Seguindo a classificação proposta pela norma do IBDF (1984), considerando simultaneamente os defeitos apresentados nessa tabela, ou seja, defeitos oriundos da superfície rolante da tora, foram enquadradas como de segunda classe as árvores de número 9, 14 e 18. As de número 15, 11, 7, 8 e 21 foram enquadradas na terceira classe e o restante das árvores foi enquadrado na quarta classe.

$\mathrm{O}$ defeito mais pronunciado apresentado pelas árvores foi a grande presença de nós e protuberâncias, esse defeito restringiu o enquadramento das árvores dentro de classes de qualidade inferior. Segundo a norma do IBDF (1984), somente podem pertencer à classe superior e primeira classe toras cujos valores são inferiores à 3\% para esta característica.

Uma observação importante é que o plantio não foi conduzido de forma adequada para gerar produtos para serraria, dessa forma, a presença intensa de nós e protuberâncias poderia ser minimizada, utilizando-se a operação de desrama. A desrama artificial é realizada com o intuito de aumentar a qualidade do produto final, obtendose madeira limpa em partes do tronco que, de outra forma, só produziriam material de qualidade inferior (VALE et al., 2002).

Cerne, Lavras, v. 19, n. 1, p. 43-49, jan./mar. 2013 
Tabela 3 - Valores médios e coeficiente de variação para alguns defeitos relativos à forma observados.

Table 3 - Mean values and coefficient of variation for some observed defects regarding the tree shape.

\begin{tabular}{ccccccc}
\hline Árvore & $\begin{array}{c}\text { Achatamento } \\
(\%)\end{array}$ & $\begin{array}{c}\text { CV } \\
(\%)\end{array}$ & $\begin{array}{c}\text { Conicidade } \\
(\%)\end{array}$ & $\begin{array}{c}\text { CV } \\
(\%)\end{array}$ & $\begin{array}{c}\text { Encurvamento } \\
(\%)\end{array}$ & $\begin{array}{c}\text { CV } \\
(\%)\end{array}$ \\
\hline 1 & 93,6 & 4,4 & 1,5 & 0,9 & 1,1 & 30,4 \\
2 & 87,6 & 7,7 & 1,8 & 0,5 & 2,5 & 85,7 \\
3 & 85,1 & 16,2 & 3,1 & 2,1 & 1,3 & 25,9 \\
4 & 80,8 & 8,5 & 1,3 & 1,4 & 3,0 & 51,8 \\
5 & 86,8 & 4,1 & 1,1 & 0,6 & 2,1 & 12,7 \\
6 & 89,3 & 3,3 & 1,5 & 0,6 & 2,8 & 51,0 \\
7 & 89,4 & 3,5 & 1,4 & 1,0 & 1,9 & 55,9 \\
8 & 88,9 & 3,9 & 1,8 & 1,0 & 3,9 & 36,4 \\
9 & 87,7 & 10,4 & 1,8 & 0,5 & 2,2 & 27,2 \\
10 & 86,7 & 8,8 & 1,4 & 0,6 & 2,4 & 49,7 \\
11 & 87,4 & 9,0 & 1,7 & 1,3 & 2,2 & 74,6 \\
12 & 85,9 & 5,8 & 1,5 & 0,5 & 3,0 & 55,5 \\
13 & 90,0 & 6,1 & 1,4 & 0,3 & 3,6 & 60,6 \\
14 & 93,6 & 5,6 & 1,9 & 1,0 & 2,5 & 31,8 \\
15 & 86,8 & 7,9 & 0,9 & 0,8 & 2,3 & 50,4 \\
16 & 88,5 & 7,5 & 1,1 & 0,4 & 3,5 & 23,9 \\
17 & 82,6 & 6,5 & 2,7 & 2,0 & 2,6 & 36,8 \\
18 & 86,6 & 1,3 & 0,9 & 1,3 & 1,3 & 45,5 \\
19 & 80,6 & 15,4 & 1,5 & 1,6 & 3,3 & 36,7 \\
20 & 87,8 & 12,0 & 1,1 & 0,7 & 2,3 & 50,8 \\
21 & 88,5 & 5,8 & 1,1 & 0,8 & 17,4 & 45,6 \\
\hline Média & 87,3 & 3,6 & 1,6 & & & \\
\hline
\end{tabular}

CV - Coeficiente de variação

Tabela 4 - Valores médios e respectivos coeficientes de variação para os defeitos de árvores de T. Ciliata.

Table 4 - Mean values and respective coefficient of variation for defects in T. ciliata trees.

\begin{tabular}{ccccccc}
\hline Árvore & $\begin{array}{c}\text { Inclinação da grã } \\
(\%)\end{array}$ & $\begin{array}{c}\mathrm{CV} \\
(\%)\end{array}$ & $\begin{array}{c}\text { Nós e protuberâncias } \\
(\%)\end{array}$ & $\begin{array}{c}\mathrm{CV} \\
(\%)\end{array}$ & $\begin{array}{c}\text { Rachaduras } \\
\text { superficiais } \\
(\%)\end{array}$ & $\begin{array}{c}\mathrm{CV} \\
(\%)\end{array}$ \\
\hline 1 & 4,5 & 83,7 & 11,5 & 74,8 & 11,4 & 69,2 \\
2 & 2,0 & 115,5 & 17,2 & 104,9 & 2,2 & 78,4 \\
3 & 1,4 & 62,8 & 22,4 & 101,1 & 6,9 & 64,4 \\
4 & 2,9 & 98,6 & 11,5 & 153,3 & 4,0 & 120,0 \\
5 & 3,1 & 81,7 & 12,7 & 91,2 & 4,7 & 75,8 \\
6 & 6,0 & 22,8 & 13,7 & 62,7 & 7,8 & 52,2 \\
7 & 4,4 & 96,8 & 7,9 & 68,4 & 1,6 & 115,5 \\
8 & 4,6 & 74,4 & 6,4 & 120,2 & 8,0 & 75,3 \\
9 & 1,0 & 76,6 & 4,7 & 152,5 & 14,6 & 31,1 \\
10 & 2,6 & 94,6 & 10,7 & 53,6 & 3,9 & 117,1 \\
11 & 2,4 & 113,1 & 10,0 & 104,3 & 3,7 & 107,1 \\
12 & 4,6 & 57,9 & 12,2 & 64,6 & 9,5 & 26,2 \\
13 & 3,3 & 68,7 & 14,7 & 108,3 & 2,8 & 86,5 \\
14 & 5,3 & 86,8 & 5,3 & 87,0 & 8,7 & 46,0 \\
\hline
\end{tabular}


Tabela 4 - Continuação...

Table 4-Continued...

\begin{tabular}{ccccccc}
\hline Árvore & $\begin{array}{c}\text { Inclinação da grã } \\
(\%)\end{array}$ & $\begin{array}{c}\text { CV } \\
(\%)\end{array}$ & $\begin{array}{c}\text { Nós e protuberâncias } \\
(\%)\end{array}$ & $\begin{array}{c}\text { CV } \\
(\%)\end{array}$ & $\begin{array}{c}\text { Rachaduras } \\
\text { superficiais } \\
(\%)\end{array}$ & $\begin{array}{c}\text { CV } \\
(\%)\end{array}$ \\
\hline 15 & 2,0 & 89,1 & 9,8 & 69,1 & 2,8 & 173,2 \\
16 & 2,3 & 162,9 & 12,9 & 94,3 & 5,0 & 111,7 \\
17 & 3,7 & 76,3 & 12,5 & 58,3 & 5,2 & 86,9 \\
18 & 3,1 & 34,7 & 6,0 & 79,8 & 4,8 & 80,0 \\
19 & 4,1 & 104,6 & 14,8 & 80,1 & 16,4 & 35,3 \\
20 & 3,8 & 73,9 & 12,1 & 49,8 & 12,7 & 99,7 \\
21 & 3,5 & 84,0 & 7,1 & 116,3 & 5,8 & 70,6 \\
\hline Média & 3,4 & 83,8 & 11,2 & 90,2 & 6,8 & 82,0 \\
\hline
\end{tabular}

Para eucalipto, o autor supracitado relatou valores de nós e protuberância inferiores a $3 \%$ para híbridos de $E$. urophylla e E. camaldulensis, mesmo sem a utilização de desrama natural.

Os defeitos de inclinação da grã e rachaduras de superfície não foram determinantes para o enquadramento das árvores nas classes inferiores. Se não fosse a consideração dos defeitos nós e protuberâncias na classificação, ou seja, considerando apenas a inclinação da grã e rachaduras de superfície simultaneamente, as árvores de número 2, 4, 10, 11, 15 e 16 seriam consideradas como de classe superior, a árvore 3 como de primeira classe, e o restante de segunda classe. Ressalta-se, portanto, que medidas e técnicas de manejo e melhoramento genético devem ser aplicadas para reduzir os defeitos de nós e protuberâncias.

A seguir, na Tabela 5, são apresentados os valores médios obtidos para a espessura da rachadura superficial.

A espessura média das rachaduras foi de $3,14 \mathrm{~mm}$ com um coeficiente de variação médio de 36,7\%. Para Eucalyptus spp., Ferreira et al. (2004) verificaram uma média de $0,97 \mathrm{~mm}$ para os clones estudados. Scanavaca Júnior e Garcia (2003) não encontraram correlações entre as rachaduras das toras com as rachaduras nas peças serradas, indicando que estas não são uma boa característica para a seleção de árvores para serraria.

Entretanto, considerando o nível médio de rachaduras de topo e coeficientes de variação apresentados, anteriormente para clones de Eucalyptus, pode-se verificar que várias árvores de $T$. ciliata apresentam resultados satisfatórios, tanto em termos de espessura de rachadura quanto dos menores valores de coeficientes de variação. Dentre essas árvores podem-se destacar, principalmente, as de número 5, 7, 13 e 18.
Tabela 5 - Valores médios da espessura da rachadura superficial.

Table 5 - Mean values of the surface crack thickness.

\begin{tabular}{ccc}
\hline Árvore & $\begin{array}{c}\text { Espessura da rachadura } \\
\text { superficial }(\mathrm{mm})\end{array}$ & $\begin{array}{c}\text { CV } \\
(\%)\end{array}$ \\
\hline 1 & 4.96 & 36,6 \\
2 & 2.43 & 37,6 \\
3 & 3.19 & 22,0 \\
4 & 1.91 & 53,8 \\
5 & 1.96 & 26,9 \\
6 & 2.94 & 49,3 \\
7 & 2.54 & 5,0 \\
8 & 3.06 & 43,4 \\
9 & 3.31 & 17,8 \\
10 & 2.37 & 28,6 \\
11 & 2.48 & 39,8 \\
12 & 5.02 & 39,8 \\
13 & 1.96 & 17,2 \\
14 & 4.73 & 48,0 \\
15 & 2.87 & 66,7 \\
16 & 2.26 & 38,5 \\
17 & 2.70 & 35,9 \\
18 & 1.81 & 20,8 \\
19 & 5.22 & 56,5 \\
20 & 5.35 & 64,4 \\
21 & 2.84 & 21,5 \\
\hline Média & 3.14 & 36,7 \\
\hline & &
\end{tabular}

\section{CONCLUSÕES}

A classificação das toras mostrou que o defeito mais expressivo foi a presença de nós e protuberâncias. $\mathrm{O}$ defeito menos pronunciado foi a conicidade. Para melhorar a qualidade das toras recomenda-se a utilização de

Cerne, Lavras, v. 19, n. 1, p. 43-49, jan./mar. 2013 
melhoramento genético aliado a técnicas de manejo, principalmente a poda.

\section{AGRADECIMENTOS}

CAPES, FAPEMIG, CNPq, Empresa BELA VISTA FLORESTAL, Consórcio APIFLOR, Instituto Estadual de Florestas (IEF).

\section{REFERÊNCIAS}

AGÊNCIA NACIONAL DE ÁGUAS. Hidroweb. Disponível em: <http://hidroweb.ana.gov.br/>. Acesso em: 4 nov. 2010.

BRISTOW, M.; ANNANDALE, M.; BRAGG, A. Growing rainforest timber trees: a farm forestry manual for north Queensland. Barton: Rirdc, 2005. 77 p. (Publication, 03/010).

BYGRAVE, F.; BYGRAVE, P. Growing australian red cedar: and other Meliaceae species in plantation. Barton: Rirdc, 2005. 60 p. (Publication, 04/135). Disponível em: $<$ http://findarticles.com/p/articles/mi_7252/is_2_69/ai_n31677 196/>. Acesso em: 5 dez. 2008.

CAIXETA, R. P.; TRUGILHO, P. F.; LIMA, J. T.; ROSADO, S. C. S. Classificação de Eucalyptus relacionados com a qualidade da madeira após a secagem natural. Scientia Forestalis, Piracicaba, n. 61, p. 49-58, jun. 2002.

EVANGELISTA, W. V. Caracterização da madeira de clones de Eucalyptus camaldulensis Dehnh. e Eucalyptus urophylla S.T. Blake, oriunda de consórcio agrossilvipastoril. 2007. 120 f. Dissertação (Mestrado em Ciência Florestal) Universidade Federal de Viçosa, Viçosa, 2007.

FERREIRA, S.; LIMA, J. T.; ROSADO, S. C. S.; TRUGILHO, P. F. Influência de métodos de desdobro tangenciais no rendimento e na qualidade da madeira de clones de Eucalyptus spp. Cerne, Lavras, v. 10, n. 1, p. 10-21, 2004. GRIFFITHS, M. W. The biology and host relations of the red cedar tip moth, Hypsipyla robusta in Australia. In: AUSTRALIAN FOREST GROWERS CONFERENCE, 9., 2000, Camberra. Proceedings... Camberra: ACIAR, 2000. p. 135-140.

GROSSER, D. Defeitos da madeira. Curitiba: FUPEF, 1980. 63 p. (Serie Técnica, 2).

HAWLEY, R. C.; SMITH, D. M. Silvicultura prática. Barcelona: Ômega, 1972. 544 p.

\section{INSTITUTO BRASILEIRO DE DESENVOLVMENTO} FLORESTAL. Norma para medição e classificação de toras de madeiras de folhosas. Brasília: Brasiliana, 1984. 42 p.

ROCHA, M. P. Técnicas e planejamento em serrarias. 5. ed. Curitiba: UFPR, 2001. 105 p.

SCANAVACA, L. J.; GARCIA, J. N. Rendimento em madeira serrada de Eucalyptus urophylla. Scientia Forestalis, Piracicaba, n. 63, p. 32-43, jun. 2003.

TIBÚRCIO, M. T. T. Processamento da madeira. Bauru: USC, 2000. 242 p.

VALE, R. S.; MACEDO, R. L. G.; VENTURIN, N.; MORI, F. A.; MORAIS, A. R. Efeito da desrama artificial na qualidade da madeira de clones de eucalipto em sistema agrossilvipastoril. Revista Árvore, Viçosa, v. 3, n. 26, p. 285297, jun. 2002. 
